\title{
Implementation of an Eta Belt Domain on Parallel Systems
}

\author{
Jules Kouatchou; Miodrag Rancic; Peter Norris ${ }^{\ddagger}$ and Jim Geiger ${ }^{\S}$ \\ NASA Goddard Space Flight Center \\ Code 931 \\ Greenbelt, MD 20771
}

\begin{abstract}
We extend the Eta weather model from a regional domain into a belt domain that does not require meridional boundary conditions. We describe how the extension is achieved and the parallel implementation of the code on the Cray T3E and the SGI Origin 2000. We validate the forecast results on the two platforms and examine how the removal of the meridional boundary conditions affects these forecasts. In addition, using several domains of different sizes and resolutions, we present the scaling performance of the code on both systems.
\end{abstract}

To be presented at The 2nd Workshop on Parallel and Distributed Scientific and Engineering Computing with Applications, Hyatt Regency San Francisco Airport, San Francisco, April 23-27, 2001.

\footnotetext{
- Jules.kouatchou.1 Ogsfc.nasa.gov, also affiliated with Morgan State University and his research was supported by NASA under the grant No. NAGS-3508.

${ }^{\dagger}$ Miodrag.rancic.1 Qgsfc.nasa.gov, also affiliated with Goddard Earth Sciences and Technology Center.

'Peter.norris.1@gsfc.nasa.gov, also affiliated with Goddard Earth Sciences and Technology Center.

${ }^{\hat{j}}$ james.v.geiger.1 $\mathrm{agsfc} . n a s a . g o v$
} 


\title{
Implementation of an Eta Belt Domain on Parallel Systems
}

\author{
Jules Kouatchou, Miodrag Rancic, Peter Norris ${ }^{\ddagger}$ and Jim Geiger ${ }^{\S}$ \\ NASA Goddard Space Flight Center \\ Code 931 \\ Greenbelt, MD 20771 \\ January 3, 2001
}

\begin{abstract}
We extend the Eta weather model from a regional domain into a belt domain that does not require meridional boundary conditions. We describe how the extension is achieved and the parallel implementation of the code on the Cray T3E and the SGI Origin 2000. We validate the forecast results on the two platforms and examine how the removal of the meridional boundary conditions affects these forecasts. In addition, using several domains of different sizes and resolutions, we present the scaling performance of the code on both systems.
\end{abstract}

\section{Introduction}

By the end of the year 2002, NASA will launch the satellite Triana. It will be the first Earth observing mission to provide a continuous, full disk view of the sunlit Earth. Two of the instruments that Triana will carry are EPIC, which will deliver science products such as total precipitable water, cloud height, aerosol index, total ozone, and a global visible cloud field image, and NISTAR, which obtains precise radiometry integrated over the entire sunlit disk. This unique set of observations has tremendous potential to aid in our understanding of the total Earth system and the effects of natural and humaninduced changes in the global environment.

As part of the HPCC Program at NASA GSFC, we have started a project (called the SunFlower Project) whose goal is to simulate some of the Triana observations and to assess the impact of Triana data for weather and climate predictions. Using the near-continuous cloud parameters observed by Triana and numerical simulation, we intend to produce a realistic climatology of full three-dimensional daily global cloud coverage.

For the simulation of the atmosphere within this project we are using the Eta model $[4,2]$. In order to compare Triana and the Eta model data on approximately the same grid without significant downscaling, the Eta model will be integrated at a resolution of about $15 \mathrm{~km}$. The integration domain (from -70 to $+70 \mathrm{deg}$ in latitude and $150 \mathrm{deg}$ in longitude) will cover most of the sunlit Earth disc and will continuously rotate around the globe following Triana. The cloud data assimilation is intended to run and produce 3D clouds on a near real-time basis. The moving domain will get its lateral boundary

- Jules.kouatchou 19gsfc.nasa.gov, also affliated with Morgan State Lniversity and his research was supported by NASA under the grant No. NAGS-3508.

${ }^{\dagger}$ Miodrag.rancic.1@gsfc.nasa.gov, also affiliated with Goddard Earth Sciences and Technology Center.

t Peter.norris.1@gsfc.nasa.gov, also affiliated with Goddard Earth Sciences and Technology Center.

${ }^{\S}$ james.v.geiger.1 1 ggsfc.nasa.gov 
conditions from a a lower resolution belt domain. Such a numerical setup and integration design is very ambitious and computationally demanding in terms of memory management, efficiency of the code and accuracy of the forecast produced.

The Eta model was originally designed for regional integration domains. Rancic et al. were able to expand the regional integration domain into a belt-like domain [6]. This belt-like domain was still seen as just a large regional domain in the sense that it used meridional boundary conditions (no periodicity). With such a choice, although producing acceptable forecasts [6], we could not meet the requirements of the SunFlower Project. The National Centers for Environmental Prediction (NCEP) subsequently released a new version of the Eta model code. We modify this code for belt domain (supposed to provide lateral boundary conditions for the moving domain in the SunFlower project) integrations. This new code, called the Eta-belt, no longer requires meridional boundary conditions, rather it incorporates periodicity. The objective of this paper is to test the efficiency of the Eta-belt in terms of both parallel performance on the Cray T3E and the SGI Origin 2000 and forecast results.

An outline of this paper is as follows. Section 2 gives a general description of the Eta weather model. Section 3 explains the strategy used to extend this model from a regional domain into a belt domain. In Section 4, we provide an overview of the two platforms. Numerical experiments appear in Section 5. We formulate some remarks and conclusions in Section 6.

\section{The Eta Model}

The Eta model [4] is a limited-area atmospheric model that serves as the major regional model at NCEP. It is a primitive-equations model based on finite differencing for the computation of atmospheric dynamics and physics under hydrostatic assumptions. The model employs the concept of "stepmountain" vertical coordinates and uses a semi-staggered horizontal distribution of variables, known as the Arakawa E-grid. Two major principles built into the model's design are: maintaining integral constraints of the continuous equations within finite-differencing approximations, and minimizing, or completely avoiding, artificial filtering of short waves. The model also utilizes a variety of sophisticated physical parameterization schemes. The Eta model is used for real-time forecasting by many groups at institutions worldwide, and it has shown remarkable skill in forecasting precipitation scores, as well as in the development and movement of severe storms $[4,5]$. For general information on the Eta model, refer to the following web site: http://www.srh.noaa.gov/ftproot/ssd/NWPMODEL/HTML/eta.htm.

The original version of the Eta model was designed and optimized for efficiency on vector based architectures. Computational demands for very fine resolution and large problem domains motivated the development of a distributed memory parallel option for the Eta model. The Eta model is parallelized using a standard two-dimensional data domain decomposition. Two- and three-dimensional data arrays containing prognostic variables (wind velocity, temperature, moisture, and pressure), as well as diagnostic and intermediate fields, are horizontally partitioned, and the resulting subdomains are distributed over the available processors (see Figure 1). Computations on the horizontal mesh use explicit time differencing.

The parallel code for the Eta model uses two types of communication: local and global. Local communications, where the data are exchanged only by neighboring processors, are typical for explicit time-differencing. Global communications involve the computations done by the master processor, which are then distributed among all processors, and are mainly used for I/O procedures within the Eta model code.

The parallel Eta code is written in Fortran 90 and uses MPI for interprocessor communications. 


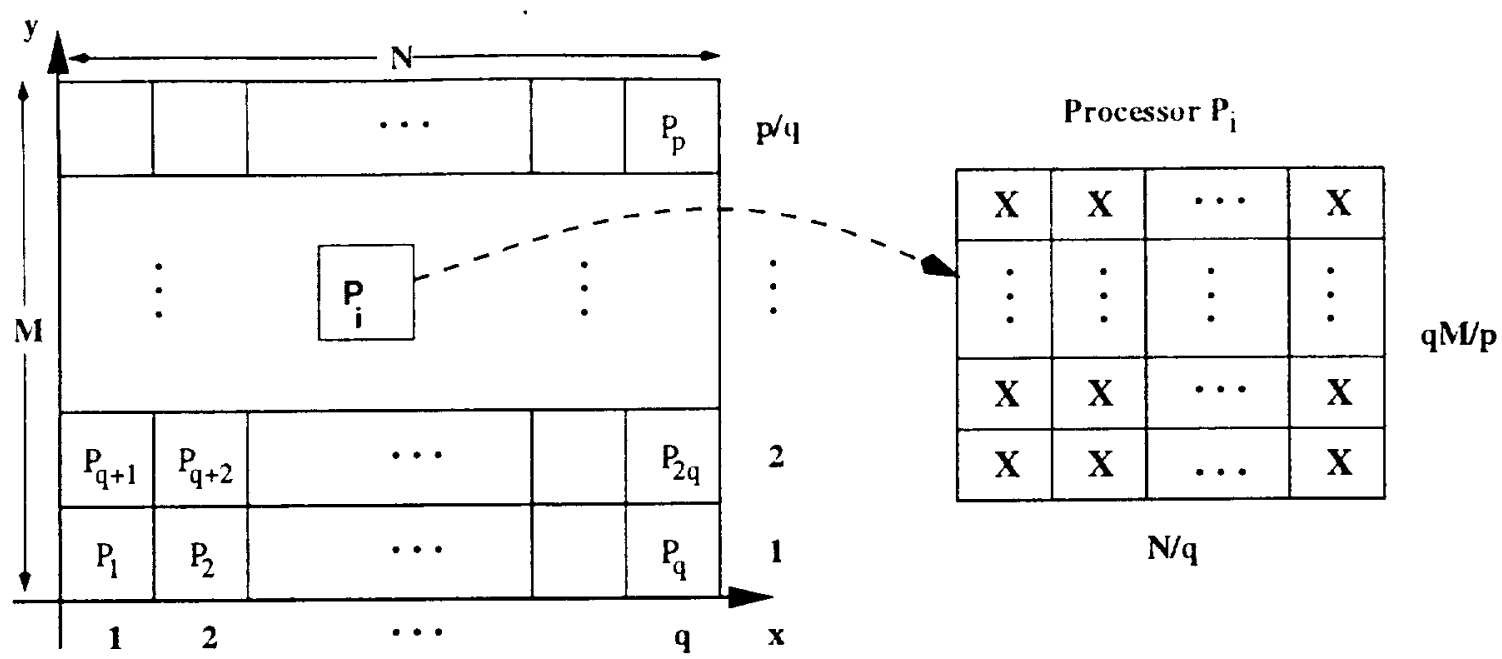

Figure 1: Domain decomposition and mapping: the $N \times M$ grid points are mapped into $p$ processors that are decomposed into a $q \times \frac{p}{q}$ array. Each processor gets $N / q \times q M / p$ grid points.

\section{Design of the Wrap-Around Belt-Domain}

In the above parallel implementation of the Eta model, it is assumed that the integration domain is a regional domain requiring zonal and meridional boundary conditions. Our objective is to extend the regional domain into a belt one where there are no meridional boundary conditions.

Rancic et al. first implemented a belt-like domain with the Eta code. This domain was a large regional one still having meridional boundary conditions. However, their implementation kept all the properties of the Eta model. In particular, their numerical experiments showed that their belt model generally produces better skill than the regional model and more significant improvement with the increase of resolution [6]. With the release of a new version of the Eta code (having improved physics, more vertical levels, etc.), we initiated an effort to design a fully periodic belt model. The idea was to modify the model integration code (on parallel computers), as well as the preprocessing and postprocessing procedures (on workstations), by stretching the regional domain in the left and right directions so that the left and right boundaries overlap.

\subsection{Preprocessing}

The preprocessing system converts global analysis data from NCEP into initial and boundary conditions for the Eta model. This system had to be adapted to provide the periodic initial and boundary conditions needed by an equatorial belt domain rather than the isolated regional domain for which it was designed. Rather than explicitly enforce periodicity throughout this lengthy code, the 360 degree belt region is extended by several degrees at either end, and these end regions are correctly loaded with input data based on the periodic requirement. With these end regions included, most of the preprocessing code is able to continue to regard the domain as an isolated region centered about the equator and prime meridian, and correct interpolation is obtained right up to the dateline without any explicit knowledge of the periodic boundary condition at this location. At output, the extended end regions are discarded so that a strictly periodic data set over 360 degrees is supplied to the model. 


\subsection{Model Integration}

The idea here is to still work with a $360^{\circ}$ domain and to incorporate the periodicity by modifying the code so that grid points along the right and left meridional boundary become neighbors (instead of isolated points). We used the regional domain version of the code and introduced changes in the domain decomposition so that each processor has right and left neighbors unlike the case with the original belt-like domain. In that case, the left-most processors marked one meridional boundary, the right-most set marked the other. In this new case, processors having portions of the domain on the left side communicate with those assigned to the right side of the domain. Figure 2 gives a representation of this decomposition strategy. In addition, some subroutines were modified (for example the one performing horizontal advection) and arrays were redimensioned to reflect this change.

Compared to the regional domain code, the new code performs a little more interprocessor communication but the same amount of computation.

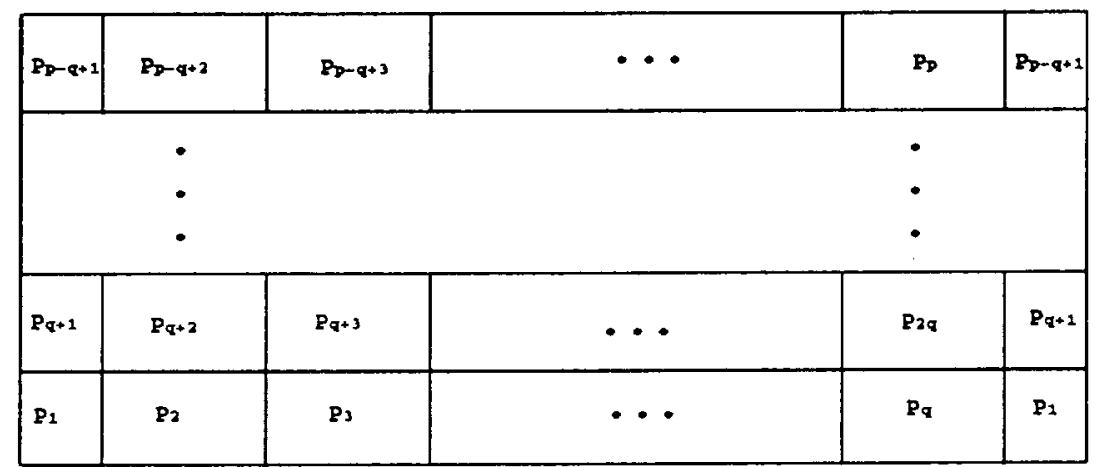

Figure 2: Domain decomposition for the belt domain.

\subsection{Postprocessing}

The postprocessing system converts output data from the Eta model into a form suitable for use with our graphical data package. This system has two stages-one to interpolate from Eta coordinate levels to standard pressure levels, and another to interpolate from the Arakawa E-grid to a regular cartesian grid. For both stages, the original isolated regional version has been modified in order to correctly perform the horizontal smoothing and interpolation procedures near the lateral periodic boundaries.

\section{Description of the Platforms}

For the set of experiments presented here, we employed two architectures: the Cray T3E and the SGI Origin 2000 (SGI O2K).

\section{Cray T3E:}

The Cray T3E is a massively parallel processor system which consists of 32 to 2048 Processors Elements (PE). Each PE is a $300 \mathrm{MHZ}$ DEC Alpha 21164 microprocessor capable of 600 million floating point operations per second and has a DRAM memory of 64 megabytes to $2 \mathrm{~GB}$. We employed a $256 \mathrm{PE}$ Cray T3E configuration located at the NASA Center for Computational Sciences at NASA Goddard Space Flight Center. 


\begin{tabular}{|l|c|c|c|}
\hline & Domain 1 & Domain 2 & Domain 3 \\
\hline \hline Coverage & $50 \mathrm{~S}-50 \mathrm{~N}$ & $70 \mathrm{~S}-70 \mathrm{~N}$ & $50 \mathrm{~S}-50 \mathrm{~N}$ \\
Resolution & $1 / 2 \mathrm{deg}$ & $1 / 2 \mathrm{deg}$ & $1 / 3 \mathrm{deg}$ \\
Grid points & $720 \times 201 \times 38$ & $720 \times 281 \times 38$ & $1080 \times 301 \times 38$ \\
Forecast length & 48 hours & 48 hours & 48 hours \\
\hline \hline
\end{tabular}

Table 1: Description of the domains.

\section{SGI Origin 2000}

We used the 512-processor SGI Origin 2000 available at the Numerical Aerospace Simulation high performance facility at NASA Ames Research Center. It is currently the largest single-image system in existence, with one operating system and a single address space. It has $192 \mathrm{~GB}$ main memory, a 2-TB FC Raid Disk Subsystem, and 327 GB disk storage. Each processor is a $400 \mathrm{MHZ}$ R12000 Processor.

Our implementation of the belt domain was first tested on the Cray T3E and then ported to the SGI O2K. The only major changes made to port the code to the SGI O2K were modifications to some system calls specific to each platform.

The Eta code requires double precision computations. On the Cray T3E, by default, each variable is declared in double precision. However, the default on the SGI O2K is single precision. To solve this problem, we introduced the compilation option $(-r 8)$ that sets all real variables to double precision and we also modified some arguments in specific MPI calls. We use general optimization levels: -03 on the Cray T3E and -02 on the SGI O2K.

\section{$5 \quad$ Numerical Experiments}

In this section, we report the results of our experiments by briefly validating our forecasts and by presenting the scaling performance of our code on both the Cray T3E and the SGI Origin 2000 (SGI $\mathrm{O} 2 \mathrm{~K}$ ). For this study, we consider three domains, which were preprocessed on an SGI workstation.

Domain 1 and Domain 3 cover the region extending from $50^{\circ} \mathrm{S}$ to $50^{\circ} \mathrm{N}$ at $1 / 2 \mathrm{deg}$ and $1 / 3 \mathrm{deg}$ resolution respectively. Domain 2 is of $1 / 2 \mathrm{deg}$ resolution and covers $70^{\circ} \mathrm{S}-70^{\circ} \mathrm{N}$ (see Table 1 ). All three domains have the same number of vertical levels (38). It is important to note that Domain 3 is 2.25 and 1.6 times as large as Domain 1 and Domain 2 respectively. For all the domains, the initial conditions were derived from the state of the atmosphere on June 10,1999 as given by NCEP's global analysis data.

We analyzed the forecast results obtained from both the Cray T3E and the SGI O2K by computing their root-mean-square differences. The differences were significantly small, and the forecasts were "identical" on the two systems.

We now briefly examine the forecasts produced by the Eta-belt. In Figure 3 we present the sea level contour plot for Domain 2 and in Figure 4 the geopotential height at $500 \mathrm{mb}$ for Domain 3, each after a 48-hour forecast period. The figures look reasonable, and the runs did not generate any spurious boundary noise. Similar results were achieved with experiments using prescribed boundary conditions for up to 15 days [1]. In addition, note that the contour lines at the left and right sides exactly match. This shows that the periodicity of our Eta-belt was properly implemented. 


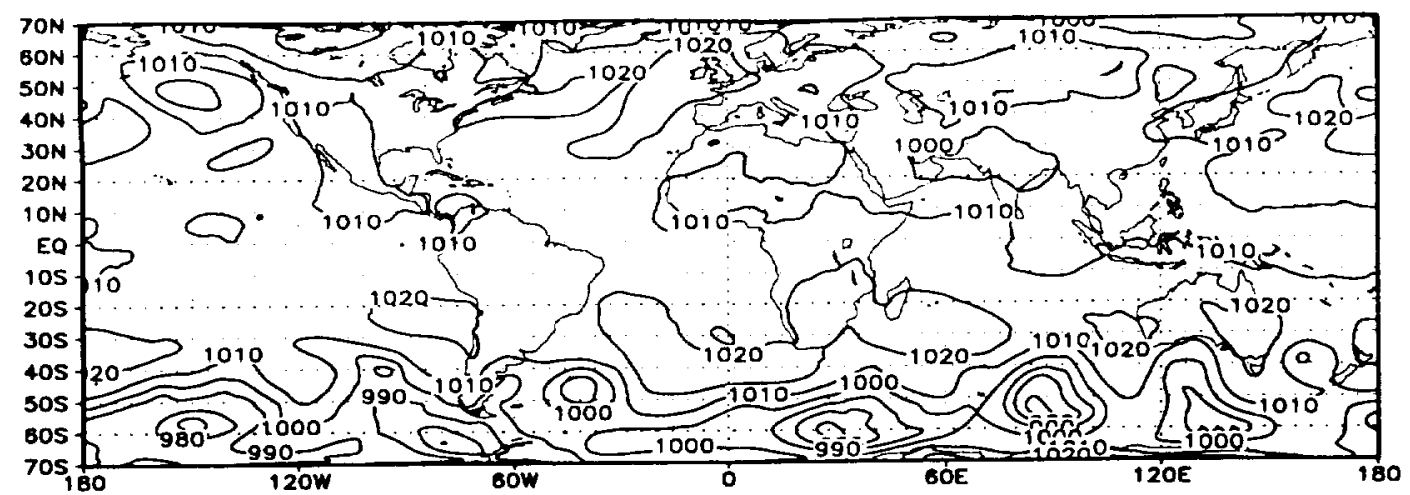

Figure 3: Domain 2: sea level pressure after a 48 hour forecast.

As it was presented in [6], we can also show that

- The forecast skill of the Eta-belt consistently improves with the increase of the horizontal resolution.

- Compared to the regional model, the Eta-belt generally has better skill and more significant improvement when the resolution is refined.

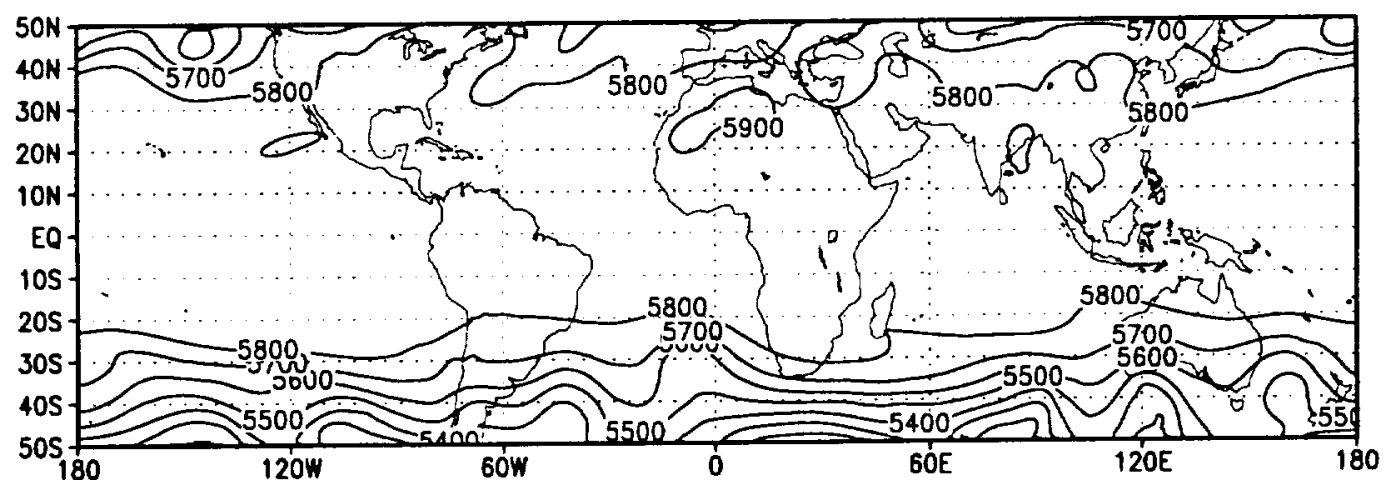

Figure 4: Domain 3: geopotential height at $500 \mathrm{mb}$ after a 48 hour forecast.

\begin{tabular}{|l|c|c||c|c|}
\hline \hline & \multicolumn{2}{|c|}{ SGI O2K } & \multicolumn{2}{c|}{ Cray T3E } \\
\hline CPUs & Time & Speedup & Time & Speedup \\
\hline \hline 64 & 1834 & 64.00 & 6426 & 64.00 \\
128 & 1066 & 110.1 & 3612 & 113.8 \\
256 & 909 & 129.1 & 2094 & 196.4 \\
\hline
\end{tabular}

Table 2: Domain 1: elapsed times and speedup as function of the number of processors.

We introduce the parallel performance of the Eta-belt by recording for the three problems the elapsed time and the speedup when the number of processors (from 6.4 to 256) varies. The results are 
reported in Table 2 , Table 3 and Table 4.

Our first remark is that when we increase the number of processors, the elapsed times decrease. The use of a belt domain does not deteriorate the parallel performance. This is consistent with results obtained with regional domains [3]. Another interesting remark is that the Eta-belt code is faster on the SGI O2K. This is due to the fact that the SGI O2K uses faster processors and carry out more efficiently interprocessor communication and $\mathrm{I} / \mathrm{O}$.

On both systems, the efficiency of the code improves as the size of the problem increases (from Problem 1 to Problem 3). Though requiring more time to complete the runs, the Cray T3E displays the best scalability. If we were to make some extrapolations, we could claim that the Eta-belt would scale beyond 512 processors on the Cray T3E. This will not necessarily be the case for the SGI O2K. In fact, we integrated Problem 3 on the SGI O2K with 320 processors and we found out that the elapsed time was about the same as the one achieved with 256 processors. Finally, because of the amount of memory available on the SGI O2K, it is possible to integrate large problems with fewer number of processors.

\begin{tabular}{|l|c|c||c|c|}
\hline \hline & \multicolumn{2}{|c|}{ SGI O2K } & \multicolumn{2}{c|}{ Cray T3E } \\
\hline CPUs & Time & Speedup & Time & Speedup \\
\hline \hline 64 & 2516 & - & N/A & - \\
128 & 1433 & 128.0 & 4917 & 128.0 \\
256 & 1096 & 167.3 & 2742 & 229.5 \\
\hline
\end{tabular}

Table 3: Domain 2: elapsed times and speedup as function of the number of processors.

\begin{tabular}{|l|c|c||c|c|}
\hline \hline & \multicolumn{2}{|c|}{ SGI O2K } & \multicolumn{2}{c|}{ Cray T3E } \\
\hline CPUs & Time & Speedup & Time & Speedup \\
\hline \hline 128 & 2226 & - & N/A & - \\
224 & 1572 & 224.0 & 4609 & 224.0 \\
240 & 1511 & 233.0 & 4257 & 242.5 \\
256 & 1508 & 233.5 & 4166 & 247.8 \\
\hline \hline
\end{tabular}

Table 4: Domain 3: elapsed times and speedup as function of the number of processors.

As for the SunFlower project (described in Section 1), we plan to use the Eta-belt with a domain extending from $70 \mathrm{deg}$ south to $70 \mathrm{deg}$ north at $1 / 3 \mathrm{deg}$ resolution (about $1080 \times 421 \times 38$ grid points). This belt domain will provide lateral boundary conditions to a regional domain continuously moving around the globe following the satellite Triana. The regional domain will extend from $70 \mathrm{deg}$ south to $70 \mathrm{deg}$ north and will cover a window of $150 \mathrm{deg}$ in longitude at $1 / 6 \mathrm{deg}$ resolution (about $900 \times 841 \times 38$ grid points). The belt and the regional domains will be integrated simultaneously in a two-way interaction mode. Such a demanding numerical setup can only be achieved on the SGI O2K (with 512 processors) because it offers a larger amount of memory and faster processors. However, we envision to use the Cray T3E for development and testing but the SGI O2K for the final Sunflower project experiments. 


\section{Conclusions}

We have expanded the Eta atmospheric model from a regional domain model into a belt domain. Our numerical experiments, carried out on the Cray T3E and the SGI Origin 2000 have shown that the new codes (on a belt domain) keeps the same forecast skill (or even does better) as the original one (on a regional domain). In addition, our forecast, results were "identical" on the two platforms. The new code runs faster on the SGI Origin 2000, but it scales better on the Cray T3E.

Acknowledgments: We would like to thank Steve Lord (the Director of EMC at NCEP) for allowing us to use the Eta code. We are grateful to Samson Cheung and Johnny Chang (of the NAS support group) for helping us implement our code on the SGI Origin 2000. Finally, we wish to thank Milton Halem, Jim Fischer (the HPCC/ESS manager) for providing computer time on the Cray T3E and the SGI Origin 2000, and Nancy Palm (the NCCS head) for accommodating our computing requirements and for providing funding support for this project.

\section{References}

[1] M. Halem, J. Kouatchou, P. Norris, M. Rancic and J. Geiger, Cloud field assimilation using Triana satellite data, Proceedings of the 33rd Cospar Scientific Assembly, Warsaw, Poland, July 16-23, 2000.

[2] Z. I. Janjic, The step-mountain eta coordinate model: further developments of the convection, viscous sublayer, and turbulence closure schemes, Mon. Wea. Rev., 126, p. 1306-1322 (1994).

[3] J. Kouatchou, M. Rancic and J. Geiger, Performance of an optimized Eta model code on the Cray T3E and a network of PCs, Proceedings of the HPCC/CAS 2000 Workshop, NASA Ames Research Center, February 15-17, 2000.

[4] F. Mesinger, Z. I. Janjic, S. Nickovic, D. Gavrilov, and D. G. Deaven, The step-mountain coordinate: model description and performance for cases of Alpine lee cyclogenesis and for a case of an Appalachian redevelopment, Mon. Wea. Rev., 116, 1493-1518 (1988).

[5] F. Mesinger, Comparison of quantitative forecasts by the 48- and by the 29-km Eta model: an update and possible implications, preprint 12 th Conf. on Numerical Weather Prediction, Phoenix, AR, Amer. Meteo. Soc., p. J22-J23 (1998).

[6] M. Rancic, J. Geiger, J. Kouatchou and F. Mesinger, A belt version of the Eta model: preliminary results, preprint 13th Conference on Numerical Weather Prediction, Denver, CO, Amer. Meteo. Soc. (1999). 